\title{
Measurement of GT exhaust gas temperature by acoustic pyrometry: preliminary error investigation
}

\author{
Gianluca Caposciutti, Lorenzo Ferrari* \\ Department of Energy, Systems, Territory and Constructions Engineering, University of Pisa \\ Largo Lucio Lazzarino 1, Pisa 56122, Italy
}

\begin{abstract}
Acoustic pyrometry is an interesting technique that may find several useful applications in turbomachinery. It is well known that the speed of sound in a medium is directly related to its temperature. Acoustic pyrometry estimates the temperature of a gas by considering the time of flight of an acoustic wave moving through it. If one acoustic emitter-receiver couple is used, only the average temperature along the acoustic path can be determined. If multiple emitter-receiver couples laying on the same plane are used, a reconstruction of the temperature map in the section is possible. In this last case, the analysis is based on the fact that the temperature of each sub portion of the section affects the time of flight of all the acoustic paths travelling across it. Many parameters affect the accuracy of the measurement. They are mainly related to the physic of the sound propagation in a medium, the accuracy of the instrumentation used, the interaction between the acoustic wave and the flow velocity and the hardware set-up. In this study, the impact of the measurement set up of an acoustic pyrometry for the measurement of the exhaust gas temperature in a gas turbine was investigated to determine the optimal solution in terms of accuracy and robustness to uncertainties.
\end{abstract}

\section{Introduction}

Gas temperature measurement has a crucial role in the operation of several devices, such as boilers, heat recovery devices, combustion chambers and turbomachinery. In gas turbines, temperature estimation is particular important as it can be used both for performance and diagnostic purposes. For instance, the comparison between the measured and expected temperature maps of exhaust gas can provide significant information on the combustion and the health of gas turbine components. Typically, in these applications, gas temperature measurement is performed by using intrusive sensors. These devices require the exposure to the gas of the sensing element, thus offering a limited resistance in harsh environments [1]. In addition, these devices interact directly with the fluid, potentially leading to an alteration of its local physical properties (e.g. local velocity or temperature). Furthermore, the necessity of achieving a thermal equilibrium with the medium leads to limitations on their size and weight in the case that dynamic measurements are requested. The use of contactless measurement techniques can have several advantages in temperature measurement, such as

\footnotetext{
* Corresponding author: lorenzo.ferrari@unipi.it
} 
a limited intrusiveness and a negligible sensing element degradation due to the reduced interaction with the medium. Among the contactless techniques for temperature measurement, an increasing interest has been devoted to acoustic pyrometry (AP). This technique allows the estimation of the temperature distribution over a given domain by analysis the sound waves propagation in the medium. In particular, this method is based on the measurement of the time of flight (TOF) of a sound wave between several emitters (i.e. speakers) and receivers (i.e. microphones). Through a specific computational procedure, the TOFs of an acoustic wave along different sound paths are used to determine the average speed of sound on a sub region of the domain, and hence the local temperature. The measurement of a TOF may be a difficult task as it is related to the domain size and the local temperature. In particular, this technique is particularly suitable for medium temperatures and large spatial domains [2], [3]. Acoustic pyrometry was successfully used by Kong et al. [4] to reconstruct the 3D temperature in a laboratory scale furnace, and they highlighted that the physical model and the emitter-receiver positioning have a significant influence on the accuracy of the temperature estimation. The temperature profile inside an industrial boiler was investigated by Bramanti et al. [5] via AP, with fair results in comparison to the measurements performed with traditional sensors. The discrepancies between the two estimations were mainly tributed to the low amount of data available for the comparison. The main parameters influencing the reliability of an AP measurement are seldom investigated in the literature. For instance, Otero et al. [6] investigated the impact of several measurement parameters on the estimation accuracy of temperature and velocity fields in a subsonic flow. In particular, they found that the emitter and receiver number has the main influence on temperature reconstruction resolution, accuracy and computational cost. This fact was also highlighted by Ferrari et al [7], which showed the influence of the number of emitters and receivers and the computational domain partitioning on the accuracy of a $2 \mathrm{D}$ temperature profile reconstruction with an acoustic pyrometry technique.

Many of the uncertainty sources in the use of AP can be tributed to errors in time of flight estimation. In this study, a simulation based on a significant case study (gas turbine exhaust gas temperature map) is used to assess the effect of the inaccuracy in TOF estimation on the reconstruction of the temperature map. In particular, a gaussian error and a uniformly distributed error are injected in TOF estimation. The error is propagated to the temperature estimation, and the performance of the AP in reconstructing the thermal map are investigated.

\section{Method}

\subsection{Temperature reconstruction method}

If an ideal gas is considered, a simple relation between the speed of sound $a$ and the temperature $T$ of a medium may be considered (Equation 1):

$$
a^{2}=\left.\frac{\partial P}{\partial \rho}\right|_{S}=k R T
$$

In Equation 1, $P, \rho$ and $S$ are the gas pressure, density and entropy, respectively. The parameter $k$ is the ratio between the constant pressure and constant volume specific heats, $R$ is the gas constant, and $T$ is the medium temperature. For the sake of simplicity, $k$ and $R$ are assumed as 1.4 and $287 \mathrm{~J} \mathrm{~kg}^{-1} \mathrm{~K}^{-1}$ (i.e. air is assumed as a medium). Several emitters $E$ and receivers $R$, are placed at the border of a square domain. Given an emitter - receiver couple (ERC), it is assumed that a sound wave propagates from the emitter to the receiver along their connecting path in a straight way. The distance between the $j$-th couple of ERCs is $L_{j}$, thus 
forming the $j$-th path. Therefore, the average speed of sound $a_{j}$ over the $j$-th path can be calculated according to Equation 2.

$$
a_{j}=\frac{L_{j}}{T O F_{j}}
$$

A computational procedure is then used to calculate the temperature profile over the square domain. The domain is ideally divided in several cells, and each $j$-th path can be decomposed along the $i$-th cells as showed by Equation 3. For the sake of simplicity, the value of the cell slowness $s_{i}$ is used instead of the cell speed of sound $a_{i}$, where $s_{i}=1 / a_{i}$.

$$
T O F_{j}=\sum_{i=1}^{N} T O F_{i}=\sum_{i=1}^{N} l_{j i} s_{i}
$$

An example of the path-lines and of the computational domain used are showed by Figure 1.

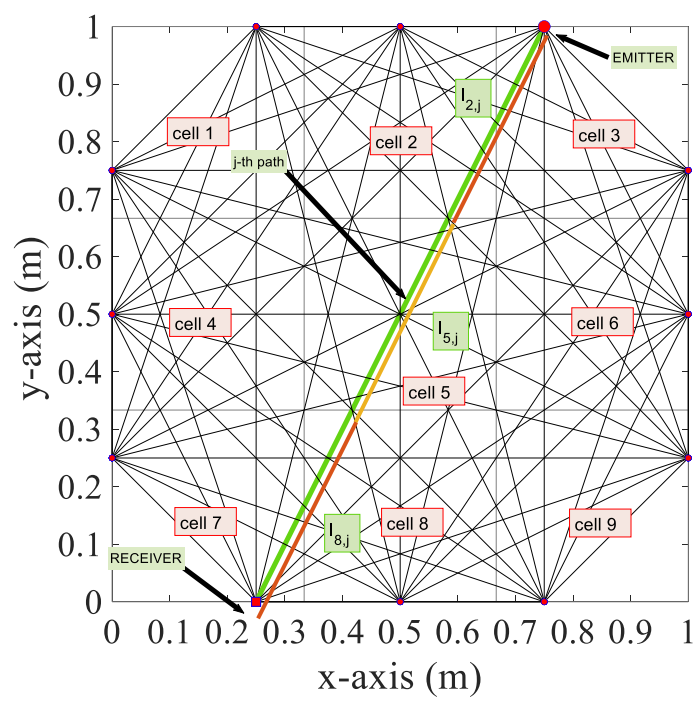

Fig.1. Example of the computational domain used: 12 ERCS and 9 cells.

Equation 3 highlights that a system of linear equations can be written with $\mathrm{N}$ variables and $\mathrm{M}$ equations, where $\mathrm{N}$ is the number of cells, and $\mathrm{M}$ is the number of paths. By solving the system, it is possible to determine the average temperature on the $i$-th cell. It can be noticed that $\mathrm{N}$ is the parameter controlling the resolution of the solution, whereas $\mathrm{M}$ is a function of the ERCs number (the combinations of ERCs taken two by two without repetitions).

The system described in Equation 3 is usually over determined, and it can be solved by means of some state-of-the-art algorithm. In particular, the SIRT algorithm is used here with satisfactory results [8]. The algorithm operation is based on an iterative procedure. At first, a solution of Equation 3 to provide $s_{i}{ }^{(k)}$ is randomly assumed and a set of tentative time of flights $\mathrm{TOF}_{\mathrm{j}}{ }^{(\mathrm{k})}$ is calculated. These values are compared with the measured TOFs, and a slowness error $\Delta s_{i}^{(k)}$ at the $k$-th iteration is estimated according to Equation 4.

$$
\Delta s_{i}^{(k)}=\left(\sum_{i=1}^{N} l_{j i}\right)^{-1} \sum_{i=j}^{M} l_{j i} \frac{T_{0 F}-T O F_{j}^{k}}{\sum_{i=j}^{M} l_{j i}}
$$


From the correction of the slowness matrix from the initial guess values, the new $s_{i}{ }^{(k+1)}$ can be calculated by summing $\Delta s_{i}{ }^{(k)}$ and $s_{i}{ }^{(k)}$. The iteration proceeds until some convergence criteria based on the absolute and relative errors are reached. In the current case, around 150 iterations are required to reach an error on time of flight estimation below $10^{-14} \%$, by spending around $2 \mathrm{~ms} /$ iteration.

\subsection{Case study}

A benchmark temperature map is used to estimate the measured time of flight. In particular, the profile is taken from De Silva et al. [9] and adapted to a square domain. The temperature values are consistent with an exhaust gas temperature map of a gas turbine, and ranges between $550{ }^{\circ} \mathrm{C}$ and $750{ }^{\circ} \mathrm{C}$. The temperature map assumed as a case study is showed in Figure 2 .

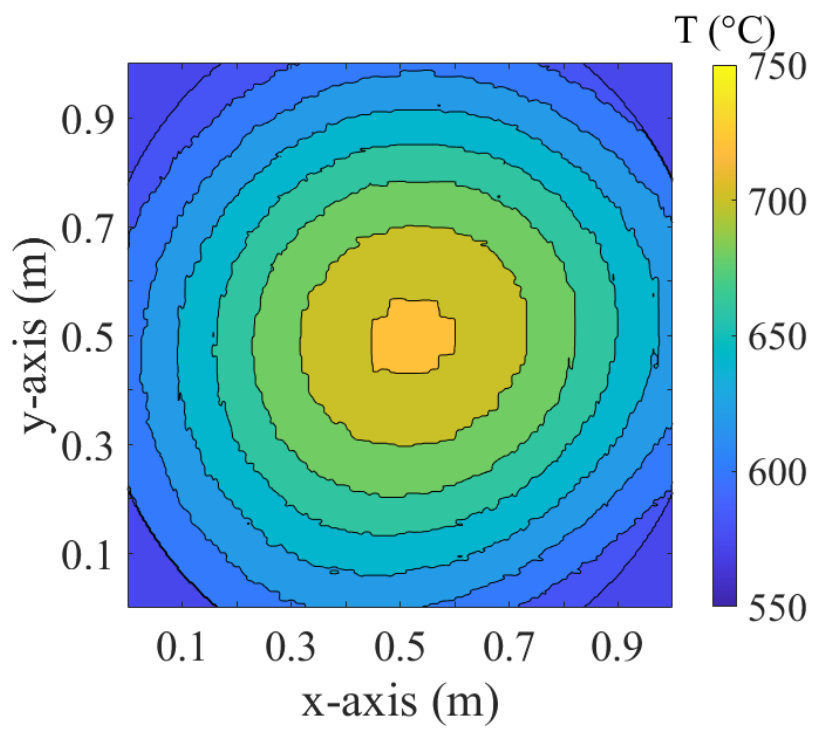

Fig.2. Temperature profile used for simulating the TOF acquisition.

The map showed in Figure 2 has a parabolic trend with the maximum temperature at the centre of the domain, but no symmetries can be observed. The values of the measured $\mathrm{TOF}_{\mathrm{j}}$ can be calculated by inverting Equation 3, thus proving a set of realistic TOFs for the analysis of the robustness to errors of the method.

\subsection{Error injection strategy}

A temperature map reconstruction via acoustic pyrometry is subjected to several sources of error. For instance, the flow speed may deflect the sound path trajectories, ERCs positioning errors can lead to a misevaluation of $l_{j i}$ and fluid inhomogeneity and instrumental inaccuracy can reduce the TOF estimation accuracy. In a previous work [10], the authors considered all these sources as included in the TOF estimation, and they cautionary considered those errors through a uniformly distributed error injection as a percentage of the measured TOF.

In the current work, a more accurate estimation is made by considering the sum of a gaussian error and a uniformly distributed error. The gaussian term considers all the unpredictable sources affecting the TOF estimation within a certain standard deviation around the TOF 
value. In addition, a uniformly distributed error with a range independent from the TOF value is used. In particular, the last source of error may reproduce better the errors related to the hardware used for the TOF estimation (digital). Therefore, the value of the new set of TOF ${ }^{*}$ affected by the error injection is calculated according to Equation 5.

$$
T O F^{*}=G N(T O F, \sigma)+R N D(\alpha)
$$

In particular, GN is a gaussian distribution centered on the TOF with standard deviation $\sigma$, and RND is a uniform distribution within $\pm \alpha / 2$ range. The literature regarding AP experiments estimates the overall accuracy of this technique within 5.4\% [11]. Bramanti et al. [5] used AP to estimate the temperature map in a $6 \mathrm{~m}$ x $5 \mathrm{~m}$ boiler section when different noise levels were introduced. They measured a TOFs in the order of $10 \mathrm{~ms}$ with a standard deviation from $0.06 \mathrm{~ms}$ to $0.15 \mathrm{~ms}$. They also considered a systematic error of $0.5 \mathrm{~ms}$ in their calculation. In the current case, the measured TOF ranges from $0.5 \mathrm{~ms}$ to $4 \mathrm{~ms}$. Therefore, the value of $\alpha$ is cautionary taken as $0.1 \mathrm{~ms}$, while $\sigma$ is varied from $0 \%$ to $2 \%$ and $5 \%$ of the TOF values.

Around 10.000 error injections are conducted for a given configuration, which is defined by a fixed number of ERCs and investigation cells. Totally, 25 ERCs (5 per side) are used for the calculation. The number of investigation cells is chosen to have at least 4 paths per cell, as suggested by [10], to increase the robustness of the reconstruction technique to measurement errors. Eventually, the computational domain was divided in 45 cells. The reconstructed and the case study temperature maps are compared by means of the indicator E. This indicator is calculated by considering the difference between the temperature estimated in a cell and the temperature of the original map in the same sub-region, and averaging all values over all the trials for that configuration. It is worth highlighting that $\mathrm{E}$ estimates (via a Monte Carlo based method) how an error in TOF can affect the temperature reconstruction profile. A scheme of the procedure is showed in Figure 3.

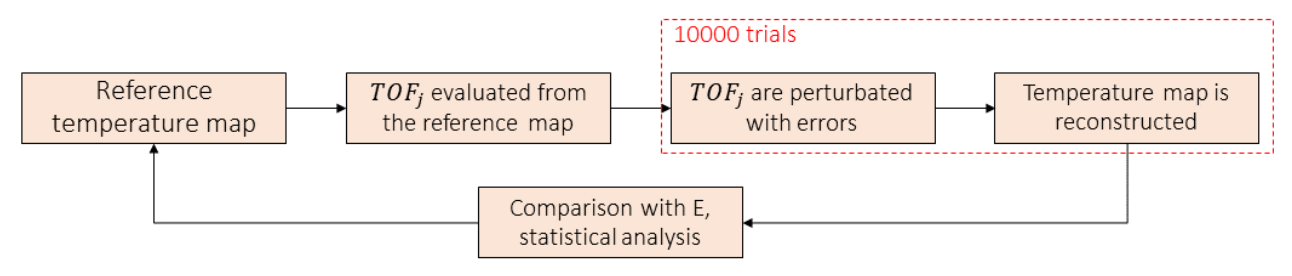

Fig.3. Scheme of the performed test

\section{Results and discussion}

The domain grid and ERCs configuration adopted in the analysis are showed in Figure 4a. Figure $4 \mathrm{~b}$ and $4 \mathrm{c}$ show the temperature map in case of $\alpha=0$ and $\sigma=0$, and the values of $\mathrm{E}$, respectively. The resulting distribution showed in Figure 4 (c) is asymmetric due to the asymmetry in the case study temperature map (see Figure 2). When no uncertainty on TOF estimation is considered, the error $\mathrm{E}$ is mainly given by the domain resolution: the greater the number of cells, the lower the value of E. By increasing the number of cell, the error is reduced, the only limit is having at least one sound path per cell, thus providing enough 
physical information to relate the TOF and the speed of sound to the cell temperature. Figure 5 shows the temperature profile reconstruction and error $\mathrm{E}$ for different values of $\sigma$ and $\alpha$.

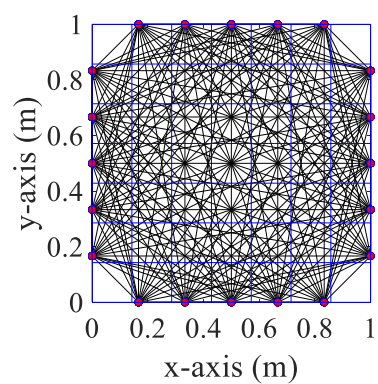

(a)

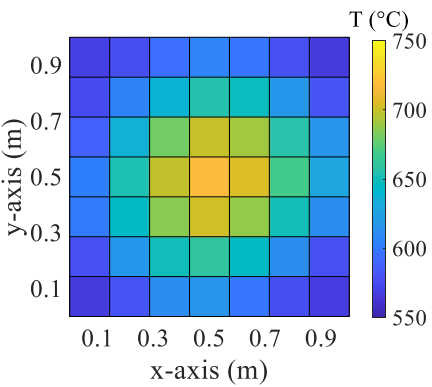

(b)

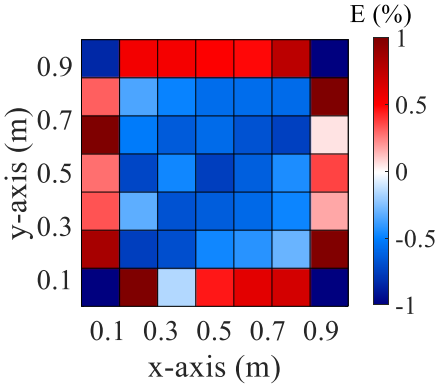

(c)

Fig.4. Domain used for calculation with cells highlighted in blue (a), temperature map reconstruction with no error injection (b), and its map of $\mathrm{E}(\mathrm{c})$.

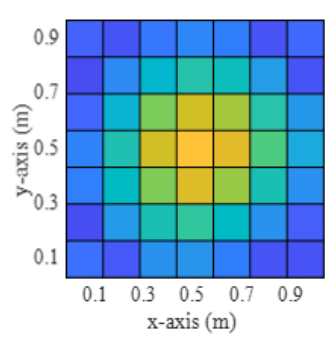

(a)

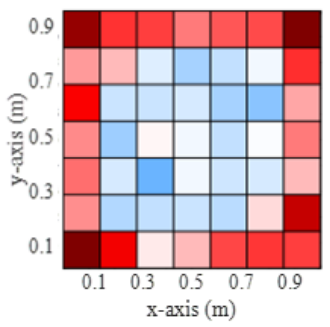

(e)

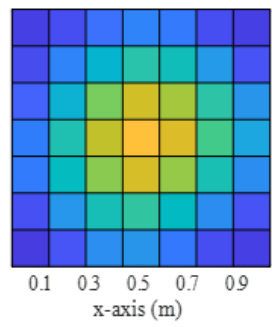

(b)

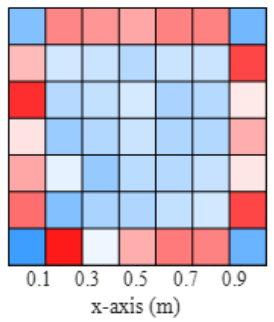

(f)

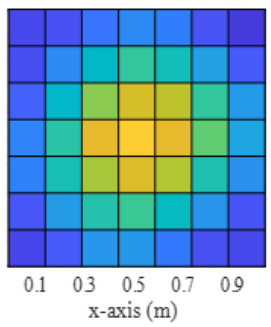

(c)

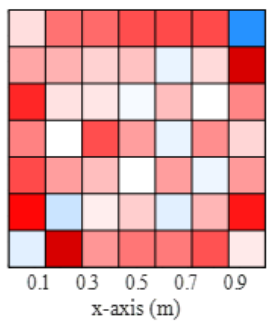

(g)

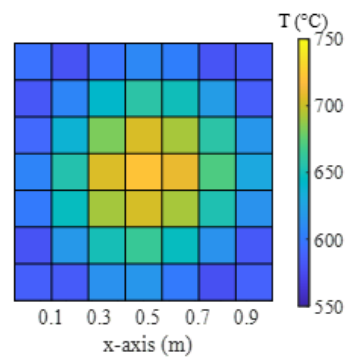

(d)

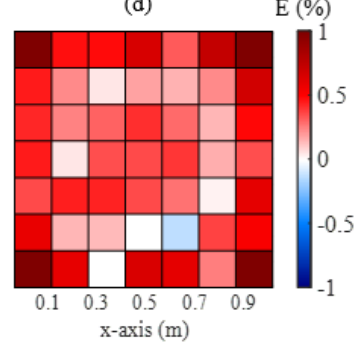

(h)

Fig. 5. Temperature profile reconstruction and related error $\mathrm{E}$ for $\alpha=0.1 \mathrm{~ms}$ and $\sigma=0(\mathrm{a}, \mathrm{e}), \alpha=0$ and $\sigma=2 \%(\mathrm{~b}, \mathrm{f}), \alpha=0$ and $\sigma=5 \%(\mathrm{c}, \mathrm{g}), \alpha=0.1 \mathrm{~ms}$ and $\sigma=5 \%(\mathrm{~d}, \mathrm{~h})$.

Figure 5 shows that for relatively small errors, being $\alpha=0.1 \mathrm{~ms}$ and $\sigma 2 \%$ and $5 \%$ in Figure $5(\mathrm{a}, \mathrm{e})$ and Figure $5(\mathrm{~b}, \mathrm{f})$ respectively, the value of $\mathrm{E}$ is within $\pm 3 \%$ and mainly located at the domain border. The value of $\mathrm{E}$ is greater where the cells have paths with a lower length. Indeed, a lower path length leads to lower TOFs, which are affected by $\alpha$ by a larger extent. Figure 6 shows the number of sound path per cell over the domain and the TOF distribution over the trials for the case with $\sigma=5 \%$ and $\alpha=0.1 \mathrm{~ms}$. 


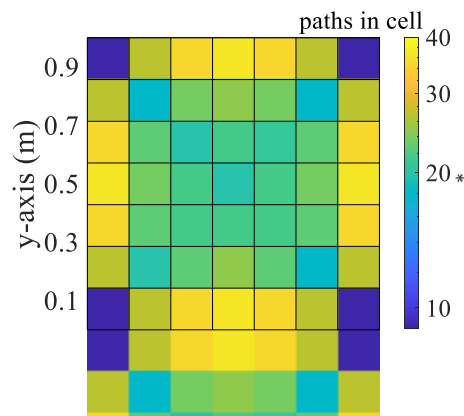

(a)

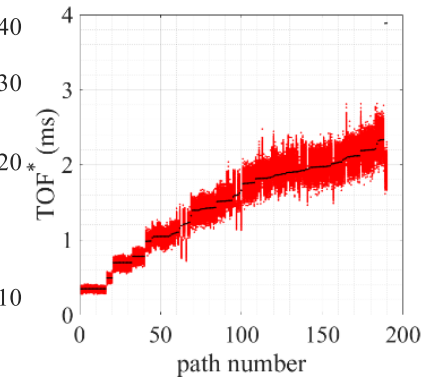

(b)

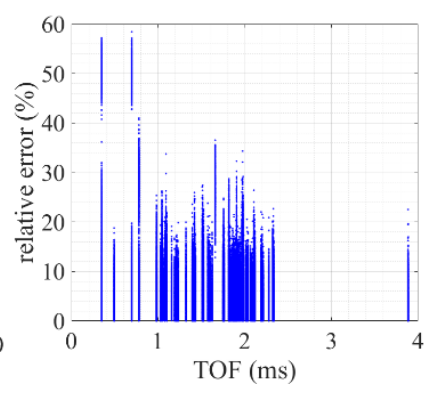

(c)

Fig. 6. Path per cell distribution (a), TOF* over the path elements, sorted from smallest to largest (b), and relative error distribution obtained by error injection on each TOF (c) for a case with $\sigma=5$ $\%$ and $\alpha=0.1 \mathrm{~ms}$.

Figure 6 (a) shows that the errors are mostly located where the number of path per cell is lower i.e. at the domain corner. However, it is clear from Figure 6 (b) and (c) that lower TOFs are affected by $\alpha$ by a larger extent. Therefore, particular attention should be paid to reduce the error sources which are not dependent on the TOF value, such as the instrument accuracy or ERC mispositioning, especially with low TOF values. On the other hand, larger time of flights are more affected by gaussian errors. It is also highlighted that the temperature accuracy strongly decrease when external error sources are considered, with an increase of |E| up to 3 times in comparison to the reference case with $\sigma=0$ and $\alpha=0$ shown in Figure 4. The errors are in the order of $3 \%$, which is consistently with the literature [11]. However, it is worth to notice that even with $\alpha=0.1 \mathrm{~ms}$ and $\sigma=5 \%$ the temperature reconstruction is still consistent and in agreement with the case study presented in Figure 2.

\section{Conclusions}

Temperature measurement has a crucial role in turbomachinery for diagnostic, monitoring and control purposes. Acoustic pyrometry can be used to collect data regarding temperature map on a given domain via contactless measurement, hence reducing sensing element degradation due to a harsh environment, and not requiring thermal equilibrium with the medium. On the other hand, several factors can affect the accuracy of the temperature map reconstructions. In this study, the effect of an error injection in time of flight estimation is investigated by considering a reference case study. Attention was paid to use a consistent error source, thus considering both gaussian and uniform errors. It turned out that the error modelling lead to results which are consistent with the literature regarding acoustic pyrometry. Moreover, it is possible to significantly increase the temperature reconstruction performance by reducing the amount of short ERC paths, hence avoiding large relative errors on estimated time of flights in those regions.

\section{References}

1. A.J. Volponi. J. Eng. Gas Turbines Power. 136, (2014).

2. X. Shen, Q. Xiong, X. Shi, K. Wang, S. Liang, M. Gao. 62, (2015). 
3. W. Fan, Y. Chen, H. Pan, Y. Ye, Y. Cai, Z. Zhang. Exp. Therm. Fluid Sci. 34, (2010).

4. Q. Kong, G. Jiang, Y. Liu, J. Sun. Appl. Therm. Eng. 179, (2020).

5. M. Bramanti, E. A. Salerno, A. Tonazzini, S. Pasini, S., A. Gray. IEEE Trans. Instrum. Meas. 45, (1996).

6. R. Otero, K.T. Lowe, W.F. Ng, K.A. Silas. Meas. Sci. Technol. 30, (2019).

7. L. Ferrari, G. Caposciutti, G. Pasini, G. F. Frate, U. Desideri. XXV Biennial Symposium on Measuring Techniques in Turbomachinery, Transonic and Supersonic Flow in Cascades and Turbomachines.

8. A.C. Kak, M. Slaney, G. Wang. Med. Phys. 29, (2002).

9. U. Desilva, R. Bunce, H. Claussen. Proceedings of the ASME Turbo Expo. 4, (2013).

10. G. Caposciutti, L. Ferrari. Journal of Engineering for Gas Turbine and Power. (2021). (to be published)

11. W. Fan, Y. Chen, H. Pan, Y. Ye, Y. Cai, Z. Zhang. 46, (2013). 